\title{
DISSENSUS, DEATH AND DIVISION
}

\author{
D. Bauso, L. Giarré, and R. Pesenti
}

\begin{abstract}
The modeling of switching systems describing networks where death and duplication processes occur is described. A dissensus protocol, complementary to consensus protocol, is introduced and the convergence or divergence of the agents' state evolution is studied. We discuss some properties of the topology reached by the network when different rules of duplication and inheritance are implemented.
\end{abstract}

Keywords: Consensus Protocols, Biological Models, Impulsive networks, Hybrid systems, Switching Systems

\section{INTRODUCTION}

Recently, a great interest has been devoted to consensus problems (see, e.g., [1], [2] and the literature cited within). In a consensus problem a set of $n$ agents reach an agreement, consensus, on the value of a given set of variables, typically the ones describing the system state. To this aim each agent can exchange information only with a subset (say it neighborhood) of adjacent agents. In its simplest form the consensus problem can be modeled as an autonomous cooperative linear system where: each component $x_{i}$ of the system state can be interpreted as the state of the $i$ th agent and the dynamic matrix is a symmetric Metzler matrix such that the sum of its columns is equal to zero. Under these hypotheses, it is easy to verify that the value of the system state converges to a vector whose components are all equal to the average of the values of the initial state components.

In this work, we study a switching system ([3], [5]) that behaves in a complementary manner to the system described above. Between two consecutive switchings, it appears as an autonomous competitive linear system where, again, each component $x_{i}$ of the system state can be interpreted as the state of the $i$ th agent, but with an opposite dynamic matrix that is a symmetric Metzler matrix such that the sum of its columns is equal to zero. If no switch occurs, the system state diverges as all eigenvalues but one of the dynamic matrix are strictly positive and the remaining one is null. The system state remains bounded as at each switching time either an agent dies or duplicates. In the former case the agent is definitively removed from the system, whereas in the latter case, the agent, say it a parent agent, divides itself in two new children agents. Both the children initial

D. Bauso is with DINFO, Università di Palermo, 90128 Palermo, Italy, E-Mail: dario.bauso@unipa.it

Corresponding author L. Giarré is with DIAS, Università di Palermo, 90128 Palermo, Italy, E-Mail: giarre@unipa.it, Research supported by MURST-PRIN "Robust Techniques for uncertain systems control" and by MURST-PRIN 2007ZMZK5T "Decisional model for the design and the management of logistics networks characterized by high interoperability and information integration"

R. Pesenti is with DMA, Università di Venezia, 30123 Venezia, Italy, E-Mail: pesenti@unive.it. states and neighborhoods are functions of the parent state and neighborhood respectively.

The aim of this paper is to introduce a very simple model to study the property of the dynamics of a set of agents competing for a scarce resource. Here, the availability of the resource to each agent is directly described by the agent state. The agents that have a greater availability of the resource are stronger (in some sense they better fit the environment) and then can subtract further resource to the adjacent agents. This kind of networks arises in many different problems, such as averaging with finite capacity channels in sensor networks or the load balancing in a processor network (see [6] or [7]). We try our inspiration from biological networks, where the description of the death and duplication process (BMID) has been studied, in the modeling of the genome evolution or the protein domains, or the genetic epidemiological models have largely been studied. Here the birth and death models are usefully introduced as stochastic Markov chain processes (i.e. [9], [8], [10]). Note that in the present approach we are considering deterministic models and no probabilistic approach is taken into consideration. The present system can be useful also to model the interactions of a group of retailers shop in franchising of the same brand that are working in competition on the territory. The death of a node corresponds to the closing of the shop and the duplication corresponds of the opening of a new one.

In the following section, the problem of interest is formalized. Then in Section III some peculiar characteristics of the system evolution are described and some open problems are discussed. Finally some conclusions are drawn in Section IV.

\section{The Dissensus PRotocol}

We consider a switching system describing the evolution of a set of $n$ agents $\Gamma=\{1, \ldots, n\}$. Let a set of agents $\Gamma=\left\{1, \ldots, n\left(t_{k}\right)\right\}$ be given with the number of agents $n\left(t_{k}\right)$ function of the time instants $t_{k}$. For the easy of notation, the dependence on $t_{k}$ is omitted, e.g. we write $n$ and not $n\left(t_{k}\right)$, when there is no risk of ambiguity. Each agent exchange information only with a subset of neighbor agents. More formally, we assume that the set $\Gamma$ induces a single component graph $G=(\Gamma, E)$, called connection graph, whose edgeset $E$ includes all the non oriented couples $(i, j)$ of agents such that agent $i$ exchanges information with agent $j$. In this context, we define the neighborhood of an agent $i$ as the set $N_{i}=\{j:(i, j) \in E\}$.

Let the initial time $t_{0}=0$, the evolution between two consecutive switching time instants, $t_{k}$ and $t_{k+1}$, can be described as follows. 
Each agent $i$ has a dynamic: its state evolves on the basis of the local information implied by its and its neighbor agents' states.

$$
\dot{x}_{i}=u_{i}\left(x_{i}, x^{(i)}\right) \quad \forall i \in \Gamma \quad \text { for } t_{k} \leq t<t_{k+1}
$$

where:

- $u_{i}: \mathbb{R} \times \mathbb{R}^{n} \rightarrow \mathbb{R}$ is differentiable and, as the model describes a competitive system, $\frac{\partial u_{i}}{\partial x_{j}} \leq 0$ for $j \neq i$;

- $x^{(i)}$ is the state vector of the agents in $N_{i}$ with generic component $j$ defined as follows,

$$
x_{j}^{(i)}= \begin{cases}x_{j} & \text { if } j \in N_{i} \\ 0 & \text { otherwise. }\end{cases}
$$

Let us now describe the agents' dynamic at the switching time instants. A switching time instant occurs whenever an agent reaches a critical state, that is, its state either becomes zero or reaches a threshold $B$. More formally,

$t_{k+1}=\arg \min \left\{t \geq t_{k}: \exists i \in \Gamma\right.$ s.t. $\left.x_{i}(t)=0 \vee x_{i}(t)=B\right\}$.

Without loss of generality, we may assume that, within each interval $t_{k} \leq t<t_{k+1}$, we renumber the agents so that the agent reaching the critical state is always agent $n$. Then, at the switching time instants the system evolves as follows.

- If $x_{n}\left(t_{k+1}^{-}\right)=0$, we say that agent $n$ dies and is removed from the system. The agents in the neighborhood of $n$ inherit the connections of $n$. No other changes occur in the states and the neighborhoods of the agents. More formally: for each single agent $j \in N_{n}\left(t_{k}\right)$, let the connections inherited by $j$ be $\Lambda_{j} \subset N_{n}\left(t_{k}\right) \backslash\{j\}$, then we have

$$
\begin{aligned}
N_{j}\left(t_{k+1}\right) & =\left\{\begin{array}{l}
N_{j}\left(t_{k}\right) \cup \Lambda_{j} \quad \forall j \in N_{n}\left(t_{k}\right) \\
N_{j}\left(t_{k}\right) \text { otherwise }
\end{array}\right. \\
x_{j}\left(t_{k+1}\right) & =x_{j}\left(t_{k+1}^{-}\right) \quad \forall j \in \Gamma \backslash\{n\} .
\end{aligned}
$$

We impose the conditions that the union of all the connections inherited by all neighbors of $n$ must cover the whole set of neighbors $N_{n}\left(t_{k}\right)$, that is, $\bigcup_{j \in N_{n}\left(t_{k}\right)} \Lambda_{j}=$ $N_{n}\left(t_{k}\right)$. We also impose that $\bar{\Lambda}_{j}=\Lambda_{j} \cup\{j\}$ define a graph $\left(N_{n}\left(t_{k}\right), \mathcal{E}\right)$ with a single component, being $\mathcal{E}=\left\{(r, s): \exists j \in N_{n}\left(t_{k}\right)\right.$ s.t. $\left.r, s \in \bar{\Lambda}_{j}\right\}$.

Finally, the connection graph $G$ evolves according to the following equations:

$$
\begin{aligned}
\Gamma\left(t_{k+1}\right)= & \Gamma\left(t_{k}\right) \backslash\{n\} \\
E\left(t_{k+1}\right)= & E\left(t_{k}\right) \cup\left\{(j, i): j \in N_{n}\left(t_{k}\right) \wedge\right. \\
& \left.i \in \Lambda_{j}\right\} \backslash\left\{(j, n): j \in N_{i}\left(t_{k}\right)\right\} .
\end{aligned}
$$

- If $x_{n}\left(t_{k+1}^{-}\right)=B$, we say that the parent agent $n$ divides producing two children agents $n$ and $n+1$. The two children agents inherit the parent connections and state. More formally: let the connections inherited by agents $n$ and $n+1$ be $\Lambda_{n}, \Lambda_{n+1} \subseteq N_{n}\left(t_{k}\right) \cup\{n, n+1\}$ respectively. Then, we have

$$
\begin{aligned}
& N_{j}\left(t_{k+1}\right)=\left\{\begin{array}{l}
\Lambda_{n} \text { for } j=n \\
\Lambda_{n+1} \text { for } j=n+1 \\
N_{j}\left(t_{k}\right) \text { otherwise }
\end{array}\right. \\
& x_{j}\left(t_{k+1}\right)=\left\{\begin{array}{l}
\alpha \text { for } j=n \\
\beta \text { for } j=n+1 \\
x_{j}\left(t_{k+1}^{-}\right) \text {otherwise }
\end{array}\right.
\end{aligned}
$$

where $\alpha, \beta>0$ satisfying $\alpha+\beta=B=x_{n}\left(t_{k+1}^{-}\right)$. We also impose the additional conditions that $\Lambda_{n} \cup \Lambda_{n+1}=$ $N_{n}\left(t_{k}\right)$ and either $\Lambda_{n} \cap \Lambda_{n+1} \neq \emptyset$ or $n \in \Lambda_{n+1}, n+1 \in$ $\Lambda_{n}$. Finally, the connection graph $G$ evolves according to the following equations:

$$
\begin{aligned}
\Gamma\left(t_{k+1}\right)= & \Gamma\left(t_{k}\right) \cup\{n+1\} \\
E\left(t_{k+1}\right)= & E\left(t_{k}\right) \cup\left\{(n, i): i \in \Lambda_{n}\right\} \cup \\
& \left\{(n+1, i): i \in \Lambda_{n+1}\right\} \backslash \\
& \left\{(j, n): j \in N_{n}\left(t_{k}\right)\right\} .
\end{aligned}
$$

Given particular initial states, two or more agents can reach a critical state at the same time instant $t_{k}$. We deal with these situations as limit cases of very close events. Then, we consider that all the corresponding deaths and divisions occur at the same time instant but in (an arbitrary) sequence.

In the rest of the paper, we discuss the evolution of the system under consideration for different choices of $\Lambda_{j}, \alpha$ and $\beta$. We assume that $u_{i}(\cdot, \cdot)$ has the following linear structure

$$
u_{i}\left(x_{i}, x^{(i)}\right)=\sum_{j \in N_{i}}\left(x_{i}(t)-x_{j}(t)\right) .
$$

Then, between two switching time instants $t_{k}$ and $t_{k+1}$, the system evolves according to:

$$
\dot{x}_{i}(t)=\sum_{j \in N_{i}}\left(x_{i}(t)-x_{j}(t)\right), 0<x_{i}\left(t_{k}\right)<B, \forall i \in \Gamma .
$$

For the short of notation, when we refer to system (5), we understand a system that evolves according to state equations (5) between two consecutive switching times, and to the above described death and division rules at the switching times. Finally, we also understand that the system initial state $0<x_{i}(0)<B$, for all $i \in \Gamma(0)$.

Now, let the adjacency matrix $A=\left[a_{i j}\right]$ of a graph be defined as $a_{i i}=0$ and $a_{i j}=1$ if $(j, i) \in E$ where $i \neq j$; and the Laplacian matrix of the weighted digraph be defined as $L=\left[\ell_{i j}\right]$, where $\ell_{i i}=\sum_{j} a_{i j}$ and $\ell_{i j}=-a_{i j}$ where $i \neq j$. For an undirected graph, the Laplacian matrix is symmetric positive semi-definite. Then, the state equations (5) can be rewritten as

$$
\dot{x}=-L x, \quad 0<x\left(t_{k}\right)<B
$$

where $L=\left[l_{i j}\right]$ is the graph Laplacian of the network.

State equations (5) make agents' states diverge, we can say that the state of each agent tries to run away from its neighbor states. For this reason, we call this policy dissensus protocol as opposite to a consensus protocol, where the states try to converge to a common group decision value. 
Example 1: Fig. 1 shows an example of connection network and state evolution before the first switching time instant, that is in the interval $0 \leq t<t_{1}$, for a set of 8 agents whose initial state is $x(0)=$ $[1.5,1.0,1.1,1.2,0.5,0.8,1.3,0.9]^{T}$.

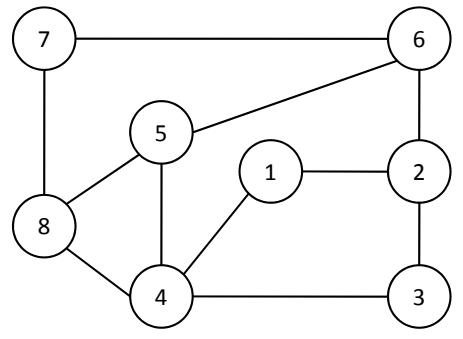

Fig. 1. An example of connection network with 8 agents.

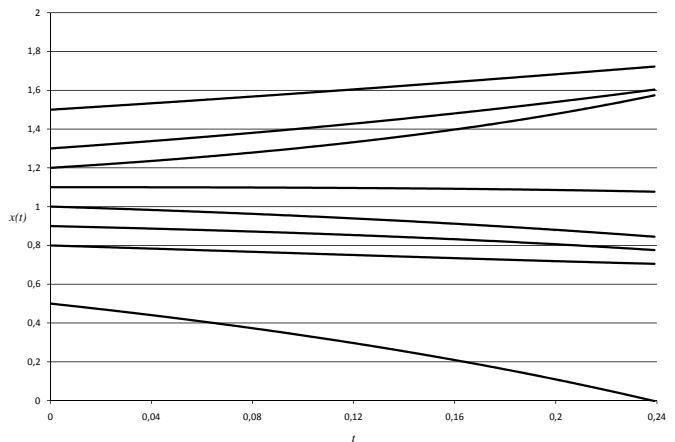

Fig. 2. The state evolution of a system of 8 agents.

In Fig. 1 and 2, we observe that agent 5 is the first one that reaches a critical state. In particular, $x_{5}\left(t_{1}^{-}\right)=0$. Then in $t_{1}$, agent 5 dies.

We conclude this section observing that we can describe the systems under consideration as a simple hybrid system. In particular, we could be interested in studying the evolution of the discrete time variable $n\left(t_{k}\right)$ that describes the number of agents alive at the switching time instants as a function of the evolution of the continuous system (5). Hereafter, we use the term alive to stress the fact that agents must have a state strictly greater than zero to be considered as an element of the system.

\section{PROPERTIES}

\section{A. Basic properties}

The first basic property is that in system (5) the sum of the components $x_{i}(t)$ of the system state is invariant:

$$
\sum_{i \in \Gamma} x_{i}(t)=\sum_{i \in \Gamma} x_{i}(0) \doteq \chi, \quad \forall t .
$$

It is apparent that $\sum_{i \in \Gamma} x_{i}(t)=\sum_{i \in \Gamma} x_{i}\left(t_{k}\right)$, for $t_{k} \leq$ $t<t_{k+1}$ as the vector $(1,1, \ldots, 1)$ is a left eigenvector, associated to the null eigenvalue, of the Laplacian matrix $L$ [4]. At a switching time instant $t_{k}$, when agent $n$ dies, its state is $x_{n}\left(t_{k}^{-}\right)=0$, whereas the states of the other agents remain unchanged. When a division occurs, the sum of the states of the children agents is equal to the state of the parent agent $x_{n}\left(t_{k}\right)+x_{n+1}\left(t_{k}\right)=x_{n}\left(t_{k}^{-}\right)$and, again, the other agents' states remain unchanged.

Here note that, differently from the case in which a linear consensus problem is implemented, condition (7) does not imply that the average value of the system state is preserved as the number of agents varies with time.

The invariance of (7) implies that, at each time, the number of agents is bounded from below according to

$$
n\left(t_{k}\right) \geq\left\lceil\frac{\chi}{B}\right\rceil, \quad \forall t_{k} .
$$

As the connectivity of graph $G$ is preserved during deaths and divisions (as it will be shown in the next paragraph), condition (8) depends critically on the values of $\alpha$ and $\beta$ in (4). We next justify the choice $\alpha+\beta=B$ by showing that if the latter does not hold true, then the number of agents converges to one or diverges to infinity. Before doing this, let us recall the notion of equilibrium for the system under consideration.

If $G$ has a single component, the system is in an equilibrium point $x^{*}$ only if all the agents have the same state, that is $x_{i}(t)=\chi / n$ for all $i \in \Gamma$. Given the matrix $L$, equilibrium $x^{*}$ is trivially unstable when there are two or more agents alive.

Now, if, for each $t_{k}, \alpha+\beta<x_{n}\left(t_{k}\right)=B$ then either the system reaches an unstable equilibrium point or $\lim _{k \rightarrow \infty} n\left(t_{k}\right)=1$. Here note that, by the system definition, the system is always in equilibrium when it includes a single agent. Differently, if, for each $t_{k}, \alpha+\beta>x_{n}\left(t_{k}\right)=B$ then either the system reaches an unstable equilibrium point or $\lim _{k \rightarrow \infty} n\left(t_{k}\right)=\infty$. With the above reasoning in mind, the choice $\alpha+\beta=B$ appears the only one that makes the model useful to describe situations different from the two critical cases discussed above.

We next expand more on the connectivity property of the division rule (4) and of the death rule (2).

Assume that $G\left(t_{k}\right)$ has a single component at time $t_{k}$ and a division occurs in $t_{k-1}$. Then, the division rule (4) preserves the connectivity of $G$. If agent $n$ is in the component of all the other agents in $t_{k+1}^{-}$, then at time $t_{k+1}$, rule (4) imposes that each agent is in the same component of either to $n$ or to $n+1$. In addition, as $\Lambda_{n} \cap \Lambda_{n+1} \neq \emptyset$ implies that $n$ and $n+1$ are both adjacent to a common agent $j$ and then are in a same component, then the transitivity property guarantees that $G\left(t_{k+1}\right)$ also has a single component.

The death rule (2) preserves the connectivity of $G$, too. Since graph $\left(N_{n}\left(t_{k}\right), \mathcal{E}\right)$ has a single component, then each pair of agents $r$ and $s$ in $N_{n}\left(t_{k}\right)$, if $(r, s) \in \mathcal{E}$, are either adjacent to a same agent $j$ or, if there exists an agent $v$ that belongs to a path from $r$ to $s$ in $\left(N_{n}\left(t_{k}\right), \mathcal{E}\right)$, they are indirectly connected being both connected to $v$. 
Generally speaking, both rules (2) and (4) are sufficient, but not necessary, conditions to preserve the connectivity of the graph $G$ at the switching time instants. As an example, in case of death, the connectivity of $G$ would be preserved even if $\Lambda_{j}=\{j\}$ for all $j \in N_{n}\left(t_{k}\right)$, if all $j \in N_{n}\left(t_{k}\right)$ are adjacent to a common agent $v \notin N_{n}\left(t_{k}\right)$. However, the two rules (2) and (4) become also necessary conditions if the connectivity must be guaranteed by agent $n$ before dying or dividing on the basis of its local information only, that is, knowing only the agents and the connections implied by the set $N_{n}$. Consider the division rule, if $\Lambda_{n} \cup \Lambda_{n+1} \neq N_{n}\left(t_{k}\right)$ then, the information locally available to agent $n$ could not guarantee that an agent $j$ in $N_{n}\left(t_{k}\right) \backslash \Lambda_{n} \cup \Lambda_{n+1}$ does not remain disconnected from the remaining ones after agent $n$ division. If $\Lambda_{n} \cap \Lambda_{n+1}=\emptyset$, then the agents in $\Lambda_{n}$ could remain disconnected from the agents in $\Lambda_{n+1}$. Similar arguments hold for the proof of the necessity of the death rule.

\section{B. Specific rules}

Let us now discuss some properties of specific division and death rules.

Initially consider the division rule which makes both children inherit all the parent connections and connect to each other. Formally

$$
\begin{aligned}
\Lambda_{n} & =N_{n}\left(t_{k}\right) \cup\{n+1\} \\
\Lambda_{n+1} & =N_{n}\left(t_{k}\right) \cup\{n\} .
\end{aligned}
$$

With such a rule, if $G(0)$ is a complete graph then $G\left(t_{k}\right)$ are complete graphs for every $t_{k}$, whatever death rule is implemented. When $G\left(t_{k}\right)$ is a complete graph, if at time $t_{k+1}$ a death occurs, no agent inherits any new connection, as each one is already adjacent to all the other agents. If at time $t_{k+1}$ a division occurs, condition (9) imposes that the two new agents are adjacent (neighbors) to each other and to all the other agents.

If rule (9) is implemented and $\alpha$ and $\beta$ are defined as follows, $\alpha=\min \left\{x_{j}\left(t_{k+1}^{-}\right): 0<x_{j}\left(t_{k+1}^{-}\right)<B\right\}, \beta=$ $B-\alpha$ then the number of agents may diverge. Consider, as an example, the evolution of a system where $n(0)=2$ and $x_{1}(0)=B / 2$ and $x_{2}(0)=3 B / 4$. By property (8), we have $n\left(t_{k}\right) \geq 2$ for all $t_{k}$. Then, at $t_{1}$ only a division can occur, obtaining $x_{1}\left(t_{1}\right)=x_{2}\left(t_{1}\right)=B / 4$ and $x_{3}(t-1)=$ $3 B / 4$. From $t_{1}$ on agents 1 and 2 are twins, in the sense that $x_{1}(t)=x_{2}(t)$ for $t \geq t_{1}$. Again, as $n\left(t_{2}\right) \geq 2$ must hold, then at $t_{2}$ only a division can occur because 1 and 2 cannot both die and a third twin is generated, indeed we have $x_{1}\left(t_{2}\right)=x_{2}\left(t_{2}\right)=x_{3}\left(t_{2}\right)=B / 8$ and $x_{4}(0)=7 B / 8$. Iterating the above argument we have that only divisions may occur and at $t_{k}$ there are $k+1$ twin agents with $x_{1}\left(t_{k}\right)=$ $x_{2}\left(t_{k}\right)=\ldots=x_{k+1}=B / 4 K$ and the $k+2$ agent with $x_{k+2}=B(1-1 / 4 K)$. Simple but cumbersome computations show that $t_{k+1}-t_{k}$ converges to zero with rate $1 / k$. This means that switching time instants $t_{k}$ exist for any $k \in \mathbb{N}$ and $\lim _{k \rightarrow \infty} t_{k}=\infty$. In turn, this implies that $\lim _{t_{k} \rightarrow \infty} n\left(t_{k}\right)=$ $\infty$.

Differently, if rule (9) is implemented and $\alpha=\beta=B / 2$ then the system may reach an equilibrium point. Note that in this case each division generates twin agents. Consider, as an example, the same system as above. At time $t_{1}$, we have $x_{1}\left(t_{1}\right)=B / 4$ and $x_{2}\left(t_{1}\right)=x_{3}\left(t_{1}\right)=B / 2$. At time $t_{2}$, the agent 1 dies (we have not renumbered the agents for the easy of exposition) and the remaining two twin agents reach an equilibrium corresponding to a state value equal to $5 B / 8$.

Until now, we have assumed the graph complete. Let us now consider the asymptotic behavior of the system with a generic connecting graph $G(0)$ when rule (9) is implemented, provided that the number of agents does not diverge and the system does not reach an equilibrium. To this aim, let us define as degree of agent $i$ the cardinality of the set $N_{i}$. Then observe that when rule (9) is implemented, at each division the degrees of the children agents are equal to the degree of the parent node plus one. The degrees of agents adjacent to the parent node increase by one, too. Whichever death rule is implemented, at each death the degree of the agents adjacent to the dying node may at maximum decrease by one.

Let us also define $\bar{n}=\lim _{\tau \rightarrow \infty} \max \left\{n\left(t_{k}\right): t_{k} \geq \tau\right\}$. If the number of agents does not diverge and the system does not reach an equilibrium, then $\bar{n}<\infty$ and $\sup \left\{t_{k}: n\left(t_{k}\right)=\right.$ $\bar{n}\}=\infty$. Consider now the generic time instant $t_{k}$ such that $n\left(t_{k}\right)=\bar{n}$, necessarily $n\left(t_{k-1}\right)=n\left(t_{k+1}\right)=\bar{n}-1$. We show that, under appropriate assumptions, either $G\left(t_{k-1}\right)$ is a complete graph or the expected value of the cardinality of $E\left(t_{k+1}\right)$ is greater than the cardinality of $E\left(t_{k-1}\right)$.

To this end, denote by $r$ and $q$ respectively the agent that divides at time $t_{k}$ and the agents that dies at time $t_{k+1}$. This denomination avoid possible ambiguities given by the renumbering of agents. Now observe that $\left|E\left(t_{k+1}\right)\right|=$ $\left|E\left(t_{k-1}\right)\right|+\left(\left|E\left(t_{k}\right)\right|-\left|E\left(t_{k-1}\right)\right|\right)+\left(\left|E\left(t_{k+1}\right)\right|-\left|E\left(t_{k}\right)\right|\right)$. If we denote by $\Delta E\left(t_{k}\right)=\left|E\left(t_{k}\right)\right|-\left|E\left(t_{k-1}\right)\right|$, we have

$$
\begin{aligned}
\Delta E\left(t_{k}\right) & =\left|N_{r}\left(t_{k-1}\right)\right|+1 \\
\Delta E\left(t_{k+1}\right) & = \begin{cases}\left|N_{q}\left(t_{k-1}\right)\right|+1 & \text { if } q \in N_{r}\left(t_{k-1}\right) \\
\left|N_{r}\left(t_{k-1}\right)\right|+1 & \text { if q child of } r \\
\left|N_{q}\left(t_{k-1}\right)\right| & \text { otherwise }\end{cases}
\end{aligned}
$$

As a consequence, if $q$ is a child of $r$ then $\left|E\left(t_{k-1}\right)\right|=$ $\left|E\left(t_{k+1}\right)\right|$, otherwise, if we can assume that the expected value of $\left|N_{q}\left(t_{k-1}\right)\right|$ is equal to the expected value of $\left|N_{r}\left(t_{k-1}\right)\right|$, the expected value of $\left|E\left(t_{k+1}\right)\right|$ is greater than the expected value of $\left|E\left(t_{k-1}\right)\right|$ unless $G\left(t_{k-1}\right)$ is complete. In this latter situation, $\left|N_{r}\left(t_{k-1}\right)\right|=\left|N_{q}\left(t_{k-1}\right)\right|$ and $r \in$ $N_{r}\left(t_{k-1}\right)$, then $\left|E\left(t_{k+1}\right)\right|=\left|E\left(t_{k-1}\right)\right|$.

Following an analogous line of reasoning we can show that the expected value of $\left|E\left(t_{r}\right)\right|$ is greater than the expected value of $\left|E\left(t_{s}\right)\right|$, if $t_{r}>t_{s}$ and $n\left(t_{r}\right)=n\left(t_{s}\right)$. This means that, asymptotically, the connecting graph of the system tends to become complete, provided an equilibrium point is not reached before.

As a word of caution, we must point out that we are not yet able to assess how reasonable is the main assumption of the previous argument, i.e, that the expected value of $\left|N_{q}\left(t_{k-1}\right)\right|$ is equal to the expected value of $\left|N_{r}\left(t_{k-1}\right)\right|$. However, our simulations seem to confirm such a result, unless very 
particular initial states were fixed. As an example, the system in Fig. 1, when rule (9) is applied and $\alpha$ is fixed equal to $\beta=B / 2$, converges to a complete graph first and then to an equilibrium point, where eight agents, each one adjacent to the other ones, all assume a state equal to 1.0375 . Here note that an equilibrium point is reached as the division rule implemented creates twins.

Let us now consider the division rule which equally divides the parent's connections between the two children. Formally,

$$
\begin{aligned}
\Lambda_{n} & =\operatorname{pick}\left(N_{n}\left(t_{k}\right)\right) \cup\{n+1\} \\
\Lambda_{n+1} & =N_{n}\left(t_{k}\right) \backslash \Lambda_{n} \cup\{n\} .
\end{aligned}
$$

Where function pick $\left(N_{n}\left(t_{k}\right)\right)$ returns a random subset of $\left\lfloor\left|N_{n}\left(t_{k}\right)\right| / 2\right\rfloor$ elements of $N_{n}\left(t_{k}\right)$. With such a rule, if $G(0)$ is a hole graph, respectively a chain graph (see Fig. 3), then $G\left(t_{k}\right)$ are hole graphs, respectively chain graph, for every $t_{k}$, whatever death rule is implemented. A graph $G\left(t_{k}\right)$ is a hole, respectively a chain graph, if its is connected and all the agents have degree two, respectively all the agents have degree two a part from two agents at the extreme of the chain whose degree is one. Assume that at time $t_{k+1}$ a death occurs, if $n$ is not an extreme of a chain, the two agents adjacent to $n$ are connected, otherwise $n$ is simply removed and no new connection is introduced. Then, if $n$ is not an extreme of a chain, the degree of the remaining agents is not changed, otherwise an agent with degree two becomes of degree one to replace the degree one of the agent just dead. Assume now that at time $t_{k+1}$ a division occurs, condition (10) imposes that, if $n$ is not an extreme of a chain, each of the two new agents are adjacent only to each others and to one of the agents adjacent to their parent, otherwise the two new agents are adjacent to each others and just one of them is adjacent to the only agent adjacent to their parent. Then the degree of the agents different from $n$ is not changed and, if $n$ is not an extreme of a chain, the two children have degree two, otherwise one of them has degree two and the other one degree one.

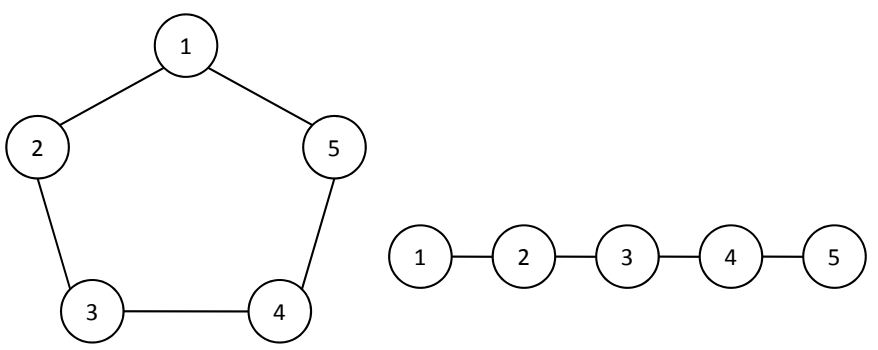

Fig. 3. A hole graph and a chain graph.

Let us now consider the asymptotic behavior of the system with a generic connecting graph $G(0)$ when the division rule (10) is implemented provided that the system does not reach an equilibrium. In particular, we assume that the death rule
(2) is, for each $j \in N_{n}\left(t_{k}\right)$, defined by

$$
\Lambda_{j}= \begin{cases}N_{n}\left(t_{k}\right) \backslash\left\{j^{*}\right\} & \text { if } j=j^{*} \\ \left\{j^{*}\right\} & \text { if } j \neq j^{*}\end{cases}
$$

where $j^{*} \in N_{n}\left(t_{k}\right)$ is arbitrarily picked. In other words, the death rule assigns all the connections of a dying agents to just one of its adjacent agents. From a practical point of view, these choices for $\Lambda_{j}$ are the simplest ones to implement that guarantee the connectivity of $G$ after the removal of the dying node.

It is immediate to see that, if at $t_{k}$ a division occurs, $\left|E\left(t_{k}\right)\right|=\left|E\left(t_{k-1}\right)\right|+1$; if at $t_{k}$ a death occurs, $\left|E\left(t_{k}\right)\right| \leq$ $\left|E\left(t_{k-1}\right)\right|-1$ as at least the connection $\left(n, j^{*}\right)$ is not substituted by a new connection. It is also apparent that the considered division and death rules forbid the creation of new cycles in the connection graph. If $\gamma\left(t_{k}\right)$ is the number of cycles present in $G\left(t_{k}\right)$ then $\gamma\left(t_{s}\right) \leq \gamma\left(t_{r}\right)$ for $t_{s}>t_{r}$.

As a consequence, if $n\left(t_{k}\right)$ diverges for $t_{k} \rightarrow \infty$, in the long run the number of divisions must exceed the number of deaths, hence $\lim _{t_{k} \rightarrow \infty}\left|E\left(t_{k}\right)\right| / n\left(t_{k}\right)=1$, i.e., the connection graph, although connected, becomes sparser and sparser. If $n\left(t_{k}\right)$ does not diverge for $t_{k} \rightarrow \infty$, we say that the density of the connection graph cannot increase in the sense that if $t_{s}>t_{r}$ and $n\left(t_{s}\right)=n\left(t_{r}\right)$ then $\left|E\left(t_{s}\right)\right| \leq\left|E\left(t_{r}\right)\right|$. Note that, a part for particular initial states or particular choices of $j^{*}$ in presence of death events, the density of the connection graph indeed decreases until $G$ presents a single or no cycle at all. The condition $\left|E\left(t_{k}\right)\right| \leq\left|E\left(t_{k-1}\right)\right|-1$ holds strictly whenever $N_{n}\left(t_{k}\right) \cap N_{j^{*}}\left(t_{k}\right) \neq \emptyset$, situation quite common if the graph is not sparse.

Our simulations show that, in general, $G\left(t_{k}\right)$ converges to a hole graph or a chain graph, but a formal proof is still missing. It is also still an open problem if rules (10) and (11) make always the system converge. The authors conjecture is that, differently from rule (9), this is always the case. The idea that supports the conjecture is that $n\left(t_{k}\right)$ may diverge if, most of the times, an agent can increase its state to reach the threshold $B$ without pushing any other agent toward a certain death. This situation may occur if the state increase of the considered agent $n$ is distributed at the expenses of the states of a sufficient number of agents adjacent or in any case not very distant from it. The agents cannot be very distant from $n$, since the state of $n$ increases with exponential speed. In case of a sparse connecting graph, on the average, each agent is close only to a limited number of other agents, then the previously described situation should not occur.

Fig. 4 reports the evolution of the system described in Section 1 when the above described division and death rules are implemented. In particular $j^{*}$ in the death rule is chosen as the agent adjacent to the dying agent with higher value of the state, $j^{*}=\arg \min \left\{x_{i}\left(t_{k+1}^{-}\right): i \in N_{n}\left(t_{k}\right)\right\}$.

\section{COnclusions}

In this paper we formalize the notion of dissensus in opposition to the well known concept of consensus. The main idea is that, given a network of dynamic agents, the state of each single agent diverges from the states of its neighbors. 


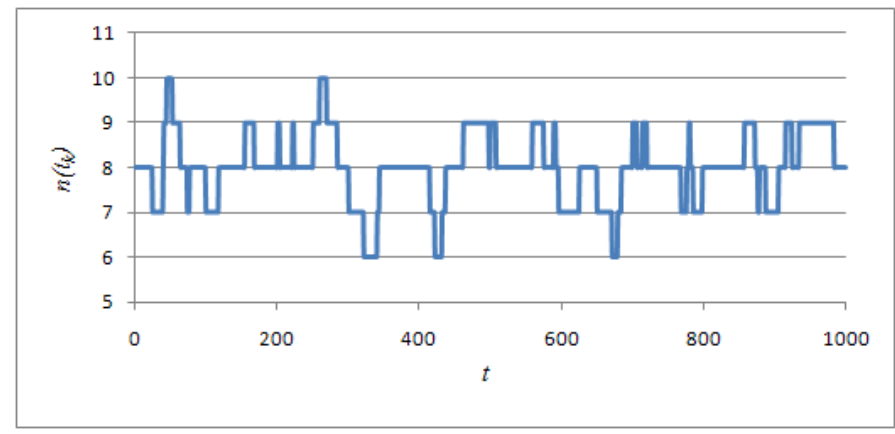

Fig. 4. Evolution of the value of $n\left(t_{k}\right)$ when rule (10) is applied.

As soon as it reaches a lower or an upper bound the agents either dies or divides in two new agents. Thus, the topology of the network is time varying and evolves according to some pre-defined rules. The aim of our current research is to look at the system as a switching/impulsive system and analyze its properties (stability, connectivity, topology) by using the tools of the switching and impulsive theory.

\section{REFERENCES}

[1] R. Olfati-Saber, J. A. Fax, and R. M. Murray. "Consensus and Cooperation in Networked Multi-Agent Systems," Proceedings of the IEEE, vol. 95, no. 1, pp. 215-233, Jan. 2007.

[2] W. Ren, R. W. Beard, and E. Atkins, "Information Consensus in Multivehicle Cooperative Control: Collective Group Behavior through Local Interaction," IEEE Control Systems Magazine, Vol. 27, Issue 2, 71-82, 2005.

[3] D. Liberzon, Switching in Systems and Control, Volume in series Systems and Control: Foundations and Applications., Birkhauser, Boston, MA, Jun 2003.

[4] D. Bauso, L. Giarré, R. Pesenti, "Nonlinear Protocols for the Optimal Distributed Consensus in Networks of Dynamic Agents", Systems and Control Letters, vol 55, no. 11, pp. 918-928, Nov. 2006.

[5] J.P. Hespanha, D. Liberzon, A.R. Teel, "Lyapunov conditions for Input to state Stability of impulsive systems", Automatica, in print 2008.

[6] A. Kashyap, T. Basar, R. Srikant, "Quantized consensus", Automatica, vol. 43, no. 7, 1192-1203, 2007.

[7] S. Boyd, A. Ghosh, B. Prabhakar, and D. Shah. Randomized gossip algorithms. IEEE Transactions on Information Theory, 52(6):25082530, 2006.

[8] Chung F, Lu L, Dewey TG, Galas DJ. Duplication models for biological network., J Comput Biol. 2003;10(5):677-87.

[9] Karev GP, Wolf YI, Rzhetsky AY, Berezovskaya FS, Koonin EV. Birth and death of protein domains: a simple model of evolution explains power law behavior, BMC Evol Biol. 2002 Oct 14;2(1):18.

[10] H. Hethcote and P. van den Driessche Some epidemiological models with nonlinear incidence, J. Math. Biol, 29:271-287, 1991. 